\title{
Optimization-based Design of Plant-Friendly Multisine Signals using Geometric Discrepancy Criteria
}

\author{
Hans D. Mittelmann* and Gautam Pendse \\ Department of Mathematics and Statistics \\ Arizona State University, Tempe, Arizona 85287 \\ Daniel E. Rivera and Hyunjin Lee \\ Control Systems Engineering Laboratory \\ Department of Chemical and Materials Engineering \\ Arizona State University, Tempe, Arizona 85287-6006
}

\begin{abstract}
System identification is an important means for obtaining dynamical models for process control applications; experimental testing represents the most time-consuming step in this task. The design of constrained, "plant-friendly" multisine input signals that optimize a geometric discrepancy criterion arising from Weyl's Theorem is examined in this paper. Such signals are meaningful for data-centric estimation methods, where uniform coverage of the output state-space is critical. The usefulness of this problem formulation is demonstrated by applying it to a linear problem example and to the nonlinear, highly interactive distillation column model developed by Weischedel and McAvoy. The optimization problem includes a search for both the Fourier coefficients and phases in the multisine signal, resulting in an uniformly distributed output signal displaying a desirable balance between high and low gain directions. The solution involves very little user intervention (which enhances its practical usefulness) and has great benefits compared to multisine signals that minimize crest factor. The constrained nonlinear optimization problems that are solved represent challenges even for high-performance optimization software.
\end{abstract}

Keywords: system identification, process control, constrained optimization

\section{Introduction}

Dynamic modeling is a critical task to many problems in the areas of simulation, prediction, and control of process systems. Given the complexity of most industrial plants, a sensible approach is to estimate dynamic models from data generated through well-designed experiments; this is the problem of system identification (Ljung, 1999). Particular industries, such as the petrochemical and refining industries, rely almost exclusively on system identification as the principal means for obtaining dynamic models for advanced control purposes.

\footnotetext{
* to whom all correspondence should be addressed; phone: (480) 965-6595, email:Hans.Mittelmann@asu.edu
}

(C) 2005 Kluwer Academic Publishers. Printed in the Netherlands.

Weylmultisinefinal3b.tex; 25/04/2005; $10: 54 ;$ p.1 
System identification is traditionally broken down into four substeps: 1) experimental planning and execution, 2) data preprocessing and model structure selection, 3) parameter estimation and 4) model validation. The quality of the data generated from the experimental design stage is critical to the success of the comprehensive system identification and subsequent control design procedures. In the chemical process industries, identification testing is by necessity conducted while the plant is in normal operation, and as such represents one of the most expensive and time consuming steps in the application of advanced control in the process industries. It comes as no surprise that model development has been reported to account for $75 \%$ of the costs associated with an advanced control project (Hussain, 1999).

The need for "plant-friendliness" in system identification for the process industries stems from the fundamental need for informative experiments despite practical requirements to the contrary (Rivera et al., 2003). A plant-friendly identification test will produce data leading to a suitable model within an acceptable time period, while keeping the changes and variability in both input and output signals within userdefined constraints. In recent years, there has been significant interest in data-centric dynamic modeling frameworks such as Just-in-Time modeling (Cybenko, 1996) and Model-on-Demand (MoD) estimation (Stenman, 1999). The appeal of these modeling approaches is that they enable nonlinear estimation, while reducing the structural decisions made by the user and maintaining reliable numerical computations. The performance of these methods, however, is highly dependent upon the availability of quality, informative databases, and consequently, good experimental designs are an imperative. An important consideration in experimental design for these estimation methods is to achieve uniform coverage of regressors in the database. This paper examines the development of multisine signal designs that are ultimately intended for data-centric frameworks and also satisfy plant-friendliness constraints during identification testing.

The idea of uniformly distributed experimental designs for system identification relying on multisine signals is not entirely new, but the concept is not well known, even within the control community. The work of Duym and Schoukens (1995) motivates the problem on the need for nonparametric identification intended for kinematic modeling applications (where both velocity and displacement output variables need to uniformly cover the state-space) as opposed to data-centric estimation. The approach relies on minimizing an objective function quantifying the real and actual discrepancy from a user-defined grid. 
An iterative procedure that does not apply constraint enforcement is used in this work.

A novel, more systematic approach that we present in this paper is to rely on the principles of geometric discrepancy theory (Matoušek, 1999) as a means for achieving uniformity of the data in a regressor space. This is accomplished by minimizing a discrepancy function made up of trigonometric polynomials arising from Weyl's Theorem that insure that the points are equidistant on a state-space. The optimization problem calls for minimizing this discrepancy function on the anticipated outputs of the system, subject to the restrictions of an orthogonal "zippered" spectrum (used to enable multi-channel implementation) and simultaneously enforcing plant-friendliness time-domain constraints on upper and lower limits, move sizes, and rates of change in either (or both) input and output signals. The optimization problem is solved using a state-of-the-art NLP solver (KNITRO 3.1) which uses an interior point trust region method and employs SQP techniques to solve the barrier subproblems. Through out the CG-iterative algorithm was chosen.

The paper is organized as follows: Section 2 presents some necessary background material, while Section 3 describes the Weyl criterion that defines the geometric discrepancy objective. Section 4 presents an example based on a simple linear highly interactive system that leads to a plant-friendly constrained optimization problem formulation that is the basis for this work. Section 5 describes the results of a more demanding case study (based on the nonlinear Weischedel-McAvoy distillation column) while Section 6 contains a Summary and Conclusions.

\section{Background}

Multisine signals are deterministic, periodic signals, which have great versatility for use in system identification. A multisine signal $u(k)$ corresponding to the $j$-th channel of a system with $m$ total inputs is represented by the equation

$$
u_{j}(k)=\sum_{i=1}^{(m+1) n_{s}} \sqrt{2 \alpha_{i j}} \cos \left(\omega_{i} k T+\phi_{i j}\right), \quad \omega_{i}=\frac{2 \pi i}{N_{s} T}, n_{s} \leq \frac{N_{s}}{2}
$$

The power spectrum in a multisine input can be directly specified by the user through the selection of the Fourier coefficients $\alpha_{i j}$, the number of independent harmonics $n_{s}$, and the signal length $N_{s} . k$ and $T$ represent 
the sampling index and sampling time, respectively. Multisine inputs are easy to implement in a real-time setting; as deterministic signals, one cycle can be designed to include all the frequency content needed for consistent estimation of the plant dynamics. Under noisy experimental conditions, multiple cycles of a periodic input can be applied until the variance in the model estimate is reduced to acceptable levels (Ljung, 1999). Multisine inputs have been deemed effective in a number of diverse application environments, from large space structures (Bayard, 1993) to industrial boilers (Godfrey, 1993).

No single criterion comprehensively defines plant-friendliness, although varied metrics have been proposed (Rivera et al., 2003). One measure that has been studied in the context of plant-friendliness is the crest factor $(C F)$ (Guillaume et al., 1991; Godfrey, 1993). The crest factor, defined as the ratio of the $\ell_{\infty}$ (or Chebyshev) norm and the $\ell_{2}$-norm of a signal $x$,

$$
C F(x)=\frac{\ell_{\infty}(x)}{\ell_{2}(x)}, \quad \ell_{p}(x)=\left[\frac{1}{N_{s}} \int_{0}^{N_{s}}|x(t)|^{p} d t\right]^{\frac{1}{p}}
$$

provides a measure of how well distributed the signal values are over its span. A low crest factor indicates that most of the elements in the input sequence are distributed near the extremum values of the sequence. For example, if two signals with equivalent power spectral densities are to be evaluated for identification purposes, the one with lower crest factor is preferred because it will deliver the same power over a lower overall span. An alternative measure of signal distribution is the Performance Index for Perturbation Signals (PIPS) (Godfrey et al., 1999)

$$
\operatorname{PIPS}(\%)=200 \frac{\sqrt{x_{r m s}^{2}-x_{\text {mean }}^{2}}}{x_{\text {max }}-x_{\text {min }}}
$$

where $x_{r m s}$ and $x_{m e a n}$ are

$$
x_{r m s}=\sqrt{\frac{1}{N_{s}} \sum_{i=1}^{N_{s}} x_{i}^{2}} \quad x_{\text {mean }}=\frac{1}{N_{s}} \sum_{i=1}^{N_{s}} x_{i}
$$

The PIPS measure ranges between 0 and $100 \%$ (compared to 1 versus $\infty$ for crest factor), which gives it an intuitive, practical appeal. 


\section{Uniform Distribution of Infinite Sequences - The Weyl Criterion}

Discrepancy theory deals with the distribution of points in space (Matoušek, 1999). The Weyl criterion (Weyl, 1916) gives the necessary and sufficient conditions for a sequence to be uniformly distributed in $[0,1)^{d}$, the d-dimensional unit interval. Suppose $u=\left(u_{1}, u_{2}, \ldots\right)$ be an infinite sequence of points in $[0,1]$. The sequence $u$ above is called uniformly distributed in $[0,1]$ if for each sub-interval $[a, b) \in[0,1]$

$$
\lim _{n \rightarrow \infty}\left(\frac{1}{n}\left|\left\{u_{1}, u_{2}, \ldots, u_{n}\right\} \cap[a, b)\right|\right)=b-a
$$

Let $f:[0,1] \rightarrow \mathbf{R}$ be a Riemann-integrable function. If the sequence $u=\left(u_{1}, u_{2}, \ldots\right)$ is uniformly distributed in $[0,1]$ then by definition of uniform distribution it is easy to see that

$$
\lim _{n \rightarrow \infty}\left(\frac{1}{n} \sum_{i=1}^{n} f\left(u_{i}\right)\right)=\int_{0}^{1} f(x) d x
$$

Equation (6) is valid for all Riemann-integrable functions. We know that any function $f(x)$ as defined above can be expanded into a complex Fourier series as follows:

$$
f(x)=\sum_{k=-\infty}^{\infty} c_{k} e^{2 \pi i k x}
$$

Here $e^{2 \pi i k x}, k=$ integer, act as basis functions for the expansion. Note that for $k=0$ the basis function is $e^{2 \pi i 0 x}=1$ for which equation (6) holds trivially. Hence for $k \neq 0$ the basis functions satisfy equation (6). This means that

$$
\lim _{n \rightarrow \infty}\left(\frac{1}{n} \sum_{j=1}^{n} e^{2 \pi i k u_{j}}\right)=\int_{0}^{1} e^{2 \pi i k x} d x
$$

But

$$
\int_{0}^{1} e^{2 \pi i k x} d x=\left(\frac{e^{2 \pi i k x}}{2 \pi i k}\right)_{0}^{1}=0
$$

From (8) and (9) and the Weirstrass approximation theorem it is easy to prove that a sequence $u=\left(u_{1}, u_{2}, \ldots\right)$ is uniformly distributed in $[0,1]$ if and only if for all integers $k \neq 0$

$$
\lim _{n \rightarrow \infty}\left(\frac{1}{n} \sum_{j=1}^{n} e^{2 \pi i k u_{j}}\right)=0
$$


This result is called the Weyl Criterion. Weyl's criterion can be extended to higher dimensions also.

THEOREM 1 (H. Weyl, 1916). A sequence $\left\{y_{1}(k), y_{2}(k)\right\}$ is equidistributed in $[0,1)^{2}$ if and only if

$$
\lim _{N \rightarrow \infty} \frac{1}{N} \sum_{k=1}^{N} e^{2 \pi i\left(l_{1} y_{1}(k)+l_{2} y_{2}(k)\right)}=0
$$

$\forall$ sets of integers $l_{1}, l_{2}$ not both zero.

Breaking the above equation into real and imaginary parts we get that the sequence $\left\{y_{1}(k), y_{2}(k)\right\}$ is equi-distributed in $[0,1)^{2}$ if and only if $\forall$ sets of integers $l_{1}, l_{2}$ not both zero the following conditions hold:

$$
\lim _{N \rightarrow \infty} \frac{1}{N} \sum_{k=1}^{N} \cos \left[2 \pi\left(l_{1} y_{1}(k)+l_{2} y_{2}(k)\right)\right]=0
$$

and

$$
\lim _{N \rightarrow \infty} \frac{1}{N} \sum_{k=1}^{N} \sin \left[2 \pi\left(l_{1} y_{1}(k)+l_{2} y_{2}(k)\right)\right]=0
$$

\section{An Illustrative Example}

To illustrate the effectiveness of the Weyl criterion for signal design, we consider a highly interactive system based on the simplified model of a high-purity distillation column (Morari and Zafiriou, 1988). The system dynamics are described in terms of the continuous time transfer function is as follows:

$$
y(s)=\frac{1}{75 s+1}\left[\begin{array}{cc}
87.8 & -86.4 \\
108.2 & -109.6
\end{array}\right] u(s)
$$

where $y(s)$ and $u(s)$ are Laplace transform of the output and input signals to the system respectively. When sampled at a $T$ sampling interval, (13) becomes

$$
\begin{aligned}
& y_{1}(k)=a y_{1}(k-1)+b\left(87.8 u_{1}(k-1)-86.4 u_{2}(k-1)\right) \\
& y_{2}(k)=a y_{2}(k-1)+b\left(108.2 u_{1}(k-1)-109.6 u_{2}(k-1)\right)
\end{aligned}
$$

where $a=e^{\frac{-T}{75}}$ and $b=(1-a)$. 


\subsection{Constrained Problem Formulation}

Our goal here is to design an input signal that is uniformly distributed and as such has good directionality information in the output state space of the system. This assumes a priori knowledge of the plant model as either an equation or a computer program that is available to the optimizer.

We introduce two cycles of input each of length $N_{s}$ and let the transients die out in the first cycle $\left(k=0, \ldots, N_{s}-1\right)$ of the output. As before the input $u(k)$ and output $y(k)$ are vectors with two components. To design a plant friendly signal we impose bound constraints on both $u(k)$ and/or $y(k)$ in the second cycle. Here, $z$ is one of $y_{1}, y_{2}, u_{1}, u_{2}$.

$$
|z(k)| \leq C_{z}, k=N_{s}, \ldots, 2 N_{s}-1
$$

In the above equations, the $C_{z}$ are user defined constants. We would also like to have the move size of $u(k)$ and $y(k)$, which is the difference between successive values in $u(k)$ and $y(k)$, to be small. We therefore impose the constraints:

$$
|z(k+1)-z(k)| \leq \Delta C_{z}, k=N_{s}-1, \ldots, 2 N_{s}-2
$$

Again the $\Delta C_{z}$ are user defined constants.

The a priori information about the plant must be available as either a model estimated from previous tests or as a computer program simulating the system. These relationships are:

$$
y_{1}(k)=f_{1}\left(u_{1}, u_{2}, y_{1}, y_{2}\right), k=0, \ldots, 2 N_{s}-1
$$

and

$$
y_{2}(k)=f_{2}\left(u_{1}, u_{2}, y_{1}, y_{2}\right), k=0, \ldots, 2 N_{s}-1
$$

Here the arguments of $f_{1}$ and $f_{2}$ indicate the dependence of $y_{1}$ and $y_{2}$ on the values of the vectors $u_{1}, u_{2}, y_{1}$ and $y_{2}$; for the example problem these correspond to (14) and (15). The inputs $u_{1}(k)$ and $u_{2}(k)$ are chosen to be multisine inputs:

$$
u_{j}(k)=\sum_{i=1}^{(m+1) n_{s}} \sqrt{2 \alpha_{i j}} \cos \left(\frac{2 \pi i}{N_{s}} k+\phi_{i j}\right), j=1,2
$$

with Fourier coefficient bounds corresponding to a modified zippered spectrum as described below:

$$
\alpha_{i j}=\left\{\begin{array}{l}
\geq 0, \quad i=j,(m+1)+j, \cdots,(m+1)\left(n_{s}-1\right)+j \\
\geq 0, \quad i=m+1,2(m+1), \cdots, n_{s}(m+1) \\
=0, \text { for all other } i \text { up to }(m+1) n_{s}
\end{array}\right.
$$


The goal is to uniformly distribute the points $\left(y_{1}(k), y_{2}(k)\right)$ in the output state space region $\left[-C_{y_{1}}, C_{y_{1}}\right) \times\left[-C_{y_{2}}, C_{y_{2}}\right)$. We wish to use the Weyl Criterion described in the previous section to achieve this uniform distribution. Since the Weyl Criterion deals with uniform distributions in $[0,1)^{2}$, we introduce a change of variables:

$$
\hat{y}_{1}(k)=\frac{y_{1}(k)+C_{y_{1}}}{2 C_{y_{1}}} \quad \hat{y}_{2}(k)=\frac{y_{2}(k)+C_{y_{2}}}{2 C_{y_{2}}}
$$

Since we only have a finite number of points, we cannot impose (11) and (12) as described before. We choose an integer $L$ and form the set $S$ as follows:

$$
S=\{x: x \in Z \text { and }|x| \leq L\}
$$

where $Z$ is the set of all integers. Then define the set $W$ as

$$
W=\left\{\left(l_{1}, l_{2}\right): l_{1} \in S, l_{2} \in S \text { and }\left(l_{1}, l_{2}\right) \neq(0,0)\right\}
$$

We then try to minimize the sum in equations (11) and (12) for all elements of the set $W$. As before we impose this "Weyl" constraint on the second cycle $\left(k=N_{s}+1, \ldots, 2 N_{s}-1\right)$ of the output. The optimization is carried out to estimate the amplitudes and phases $\alpha_{i 1}, \alpha_{i 2}, \phi_{i 1}, \phi_{i 2}, i=1, \ldots,(m+1) n_{s}$ of the $m=2$ multisine inputs. The complete problem statement is as follows:

$$
\begin{gathered}
\min _{\alpha_{i 1}, \alpha_{i 2}, \phi_{i 1}, \phi_{i 2}} t \\
\text { s.t. } \sum_{k=N_{s}+1}^{2 N_{s}-1} \cos \left[2 \pi\left(l_{1} \hat{y}_{1}(k)+l_{2} \hat{y}_{2}(k)\right)\right] \leq t, \forall\left(l_{1}, l_{2}\right) \in W \\
\text { s.t. } \sum_{k=N_{s}+1}^{2 N_{s}-1} \sin \left[2 \pi\left(l_{1} \hat{y}_{1}(k)+l_{2} \hat{y}_{2}(k)\right)\right] \leq t, \forall\left(l_{1}, l_{2}\right) \in W \\
\text { s.t. } t \geq \epsilon
\end{gathered}
$$

and subject to constraints per Equations (16)-(22). The lower bound constraint on $t$ is imposed to promote faster convergence. $\epsilon$ is chosen to be some small positive constant.

\subsection{Note on the Computer Solution}

The constrained problems designed in this paper were solved by programming them in the modelling language AMPL which has built in 
automatic differentiation up to second order derivatives. The Weyl constraints are twice continuously differentiable and so the optimizer can make direct use of second derivative information. The optimizer used was KNITRO developed by Byrd and co-workers (Byrd et al., 1999; Byrd et al., 2000). KNITRO is an interior point trust region SQP solver and is suitable for solving both large and small problems. KNITRO is available with several other interfaces, including Matlab, GAMS and Excel.

For the computations in Pendse (2004) version 3.1 of KNITRO was used and was able to solve all the optimization problems. Problem sizes could be increased somwhat for serial computations. For much larger problems, such as dozens of channels, distributed resources will be needed. Since the time of the first computations, the following codes were also applied: CONOPT-3.14D, filterSQP, IPOPT-2.2.1d, LOQO6.06, PENNLP-1.0, SNOPT-7.1-1, and version 4.0 of KNITRO. All but the first two codes were also compared in our AMPL benchmark (Mittelmann, 2004), see there for references, in which they were able to solve most of the selected problems. Here, however, the challenges seem overwhelming. SNOPT is the only code except KNITRO which succeeds in the linear example, and for the Weischedel and McAvoy distillation column case study (discussed in Section 5) only KNITRO can solve the problem. In order to further algorithm development we plan to add the AMPL scripts to our benchmark problem collection and include the results in the benchmark website cited above. Such actions have repeatedly, for example in the nonlinear and the semidefinite programming area, led to improvements by the authors of the software. To complete the algorithm references, see Drud (1994) for CONOPT and Fletcher and Leyffer (2002) for filterSQP.

\subsection{Design Variables for the Weyl Criterion}

To better understand the influence of design variables $L$ and $\epsilon$ on the distribution of points in the output state space we perform two experiments using the example problem per (13) with the bound and move sizes shown in Table I.

\subsubsection{Experiment 1}

In this experiment, we fix $\epsilon$ at a value of $10^{-3}$ and vary $L$. The distribution of points in the output state space obtained for two different simulations with $L=2$ and 6 is shown in Figure 1. Pendse (2004) contains simulations for $L=3$ through 5 . It can be seen that by increasing $L$, the uniformity in the output state space distribution 
improves dramatically. An increase in the design variable $L$ seems to move the various clusters of points at different places in the state space to achieve an approximation to a uniform distribution.

\subsubsection{Experiment 2}

In this experiment, we fix $L=3$ and vary $\epsilon$. We know from Experiment 1 that a low value of $L$ gives a relatively poor distribution. The effect of changing $\epsilon$ would be more easy to decipher on a relatively poor distribution than with a good distribution. In view of this fact we fix $L$ at a low value of 3 . Two different simulations with $\epsilon=10^{-2}$ and $10^{-6}$ are shown in Figure 2; other intermediate values can be found in Pendse (2004). We observe that a decrease in the value of $\epsilon$ has the following effect on the output state space:

1. Various clusters of points remain in more or less the same position.

2. Points within each cluster are redistributed. Decreasing the value of $\epsilon$ seems to push apart the points overall.

It should be noted that we are attempting to satisfy the Weyl criterion under certain limitations:

Limited Data: Weyl's theorem has an infinite summation; we are only using a finite amount of data in the second cycle of the system output. Given this fact, we should not expect to achieve a completely uniform distribution but only an approximation to it.

Weyl Grid Resolution: The sufficient conditions to ensure a uniform distribution (11) and (12) should hold true for all sets of integers $l_{1}$ and $l_{2}$. This enforcement is impractical and therefore we choose a finite implementation using the sets $S$ and $W$ defined in (23) and (24). There is a considerable amount of flexibility in choosing these sets. It is clear from Experiment 1 that selection of larger sets $S$ and $W$ would result in a closer approximation to equations (11) and (12) and therefore a better output space distribution. However, one should note that increasing $L$ in the set $S$ increases the size of $W$ at a rate approximately proportional to $L^{2}$. Hence from a computational point of view, one would select a reasonable value of the parameter $L$. Experiment 1 revealed that $L=6$ is a "reasonably good" value.

Tolerance Value $\epsilon$ : The Weyl criterion requires the limit on the left hand side of (11) and (12) to go to zero as $N \rightarrow \infty$. Since we have a finite amount of data we only attempt to make this sum "small enough". Again there is no straightforward way of choosing 
$\epsilon$. In Experiment 2 we saw that decreasing $\epsilon$ does not create new clusters at different locations but leads to a redistribution of points within the same cluster. Given this information, there is not much to gain by decreasing $\epsilon$ beyond a certain limit; it is much more advantageous to increase $L$ instead.

\section{Case Study: Nonlinear High-Purity Distillation Process}

Multivariable dynamical systems, in contrast to single variable ones, may be ill-conditioned and highly interactive; this phenomena presents challenges for both system identification and subsequent controller design. A demanding nonlinear and highly interactive multivariable process system that benefits from judiciously applied system identification techniques is high purity distillation (Figure 3); the methanol-ethanol distillation column model developed by Weischedel and McAvoy (1980) is commonly used as a benchmark problem (Chien and Ogunnaike, 1992; Sriniwas et al., 1995). In a typical binary high-purity distillation column (such as the Weischedel-McAvoy column), the objective is to separate a two-component mixture into streams that are very pure in one component in each of the product streams $\left(y_{D}\right.$ in the distillate $D$ and $x_{B}$ in the bottom $B$ stream). Reflux $(L)$ and boilup $(V)$ flows can be used as manipulated variables to maintain the column at desired operation. The highly interactive nature of high-purity distillation is reflected in the fact that dynamically the system will tend to respond in the principal gain direction (consisting of achieving greater purity in one stream at the expense of purity in the other) while the low gain direction (reflecting conditions where purities in both the distillate and bottom streams increase simultaneously) is much less evident. An illustration of this phenomenon for the column model per Weischedel and McAvoy (1980) using a standard zippered multisine signal design that minimizes the input crest factor $(\min \mathrm{CF}(u)$, Lee et al. (2003)) can be seen by examining the input and output state-space plots in Figure 4.

To address the demands of highly interactive systems, one approach is to modify the standard multisine signal to contain correlated harmonics with high levels of power, which improve the low gain-direction content in the data and promote better coverage of the output statespace (Lee et al., 2003) . Design parameters determined on the basis of the guidelines per Lee et al. (2003) using dominant time constant estimates $\left(\tau_{d o m}^{L}=5\right.$ and $\left.\tau_{d o m}^{H}=20 \mathrm{~min}\right)$ and user choices of $\delta=0, \alpha_{s}=2$, and $\beta_{s}=3$, lead to parameter settings of $T=2$ minutes, $n_{s}=189$,

Weylmultisinefinal3b.tex; 25/04/2005; 10:54; p.11 
and $N_{s}=378$. A value of the amplification factor $\gamma=15$ was chosen for a min $\mathrm{CF}(y)$ signal with modified spectrum; the resulting input spectrum for this signal is shown in Figure 5a. Constraints applied to the problem and salient characteristics of these signals are summarized in Table II; an output state-space plot is shown in Figure 6a.

A significant benefit of the optimization-based problem formulation presented is that nonlinear model forms can be readiliy incorporated in the design procedure, which results in an improved ability to both meet plant-friendliness requirements as well as address the directionality and uniform distribution requirements in the output for demanding applications. A polynomial Nonlinear AutoRegressive with eXternal (NARX) input model with structure as proposed by Sriniwas et al. (1995):

$$
\begin{aligned}
y(k)= & \theta^{(0)}+\sum_{i=1}^{n_{y}} \theta_{i}^{(1)} y(k-i)+\sum_{i=\rho}^{n_{u}} \theta_{i}^{(2)} u(k-i)+ \\
& +\sum_{i=1}^{n_{y}} \sum_{j=1}^{i} \theta_{(i, j)}^{(3)} y(k-i) y(k-j)+\sum_{i=\rho}^{n_{u}} \sum_{j=\rho}^{i} \theta_{(i, j)}^{(4)} u(k-i) u(k-j) \\
& +\sum_{i=1}^{n_{y}} \sum_{j=\rho}^{n_{u}} \theta_{(i, j)}^{(5)} y(k-i) u(k-j)+\ldots
\end{aligned}
$$

was estimated for the Weischedel-McAvoy column and used to generate output predictions for the optimizer in both the min $\mathrm{CF}(y)$ and Weyl-based signals design scenarios. The benefits of the Weyl-based formulation over the minimum crest factor signal design in producing a uniform distribution in the output state-space of the data can be clearly seen by contrasting Figures $6 \mathrm{a}$ and $6 \mathrm{~b}$ : the use of the Weylbased criterion clearly results in a much more uniformly distributed coverage of the state-space, and a much better suited dataset for datacentric estimation purposes. The uniform distribution of the output within the bounds specified in the problem results in a natural balance between the high and low gain information content in the data. From Table II one does notice, however, that the improvement in output state space uniformity is obtained at the cost of higher crest factor, which consequently reduces the signal-to-noise ratio of the data in a noisy data setting. As a result there is an inherent tradeoff between these objectives that needs to be recognized. One way of addressing this issue in practical input design is to include maximum crest factor bounds as inequality constraints within the Weyl problem formulation; these can be readily incorporated in the numerical optimization framework described in this paper. 
An important difference between these signal designs is observed in the input spectra (Figure 5). In the min CF (y) case, only the phases and a subset of the Fourier coefficients in the high frequency range of the multisine signal are chosen by the optimizer, while for the Weyl-based design, the optimization problem includes a search for all Fourier coefficients and phases, including those corresponding to the correlated harmonics; this can be seen in Figure 5b. Not only do these extra degrees of freedom in the optimizer contribute to the improved performance, they reduce the number of decisions made $a$ priori by the user, leading to a more practical design procedure.

\section{Summary and Conclusions}

The paper describes a constrained optimization-based formulation of th multisine input signal problem that allows users to simultaneously specify important frequency and time domain properties of these signals. The objective considered arises from the Weyl criterion, which seeks to minimize the geometric discrepancy of the output in the statespace. As a consequence, the signals are meaningful for accomplishing plant-friendly identification testing in the process industries and can be used in support of data-centric estimation algorithms. A problem formulation that helped understand design variables in the Weyl objective was shown and illustrated via a numerical example, culminating in a case study demonstrating the effectiveness of the design procedure for a high purity distillation column, a challenging nonlinear, multivariable process system.

Clearly, the power of the proposed framework lies in its flexibility, allowing the user to incorporate both linear and nonlinear models for output prediction, time-domain constraints, and information and control-theoretic frequency domain requirements. The use of state-ofthe-art interior-point optimization methods enables the efficient solution of these nonlinear and nonconvex optimization problems. Due to the nonconvexity, computed solutions can only be guaranteed to be local optima.

\section{Acknowledgments}

Funding for this work provided by grant no. PRF-37610-AC9 from the American Chemical Society-Petroleum Research Fund is gratefully 
acknowledged. We also wish to acknowledge Dr. Martin W. Braun (currently at Intel Corporation) for first bringing the concept of geometric discrepancy and the work of Matoušek (1999) to our attention.

\section{References}

Bayard, D.: 1993, 'Statistical additive uncertainty bounds using Schroeder-phased input design'. Applied Mathematics and Computation 58, 169-198.

Byrd, R., J. Gilbert, and J. Nocedal: 2000, 'A trust region method based on interior point techniques for nonlinear programming'. Mathematical Programming A(89), 149-185.

Byrd, R., M. Hribar, and J. Nocedal: 1999, 'An interior point method for large scale nonlinear programming'. SIAM J. Optim. 9, 877-900.

Chien, I.-L. and B. A. Ogunnaike: 1992, 'Modeling and control of high-purity distillation columns'. In: 1992 AIChE Annual Meeting. Miami Beach, FL. paper 2a.

Cybenko, G.: 1996, 'Just-in-time learning and estimation'. In: S. Bittani and G. Picci (eds.): Identification, Adaptation, Learning, NATO ASI. Springer, pp. 423-434.

Drud, A. S.: 1994, 'A Large Scale GRG Code'. ORSA Journal on Computing 6, $207-216$.

Duym, S. and J. Schoukens: 1995, 'Design of Excitation Signals for the Restoring Force Surface Method'. Mechanical Systems and Signal Processing 9(2), 139-158.

Fletcher, R. and S. Leyffer: 2002, 'Nonlinear Programming without a penalty function'. Math. Programming 91, 239-269.

Godfrey, K. (ed.): 1993, Perturbation Signals For System Identification. Hertfordshire, UK: Prentice Hall International (UK) Limited.

Godfrey, K., H. Barker, and A. Tucker: 1999, 'Comparison of perturbation signal for linear system identification in the frequency domain'. IEE. Proc. Control Theory Appl. 146, 535 .

Guillaume, P., J. Schoukens, R. Pintelon, and I. Kollár: 1991, 'Crest-factor minimization using nonlinear Chebyshev approximation methods'. IEEE Trans. on Inst. and Meas. 40(6), 982-989.

Hussain, M.: 1999, 'Review of the applications of neural networks in chemical process control-simulation and on-line implementation'. Artificial Intelligence in Engineering 13(1), 55-68.

Lee, H., D. Rivera, and H. Mittelmann: 2003, 'Constrained minimum crest factor multisine signals for plant-friendly identification of highly interactive systems'. In: Proc. of the 13th IFAC Symposium on System Identification (SYSID 2003). Rotterdam, The Netherlands, pp. 947-952.

Ljung, L.: 1999, System Identification: Theory for the User. New Jersey: PrenticeHall, 2nd edition.

Matoušek, J.: 1999, Geometric Discrepancy: An Illustrated Guide. Berlin: SpringerVerlag.

Mittelmann, H.: 2004, Benchmarks for Optimization Software. http://plato.asu.edu/bench.html.

Morari, M. and E. Zafiriou: 1988, Robust Process Control. Englewood Cliffs, NJ: Prentice-Hall. 


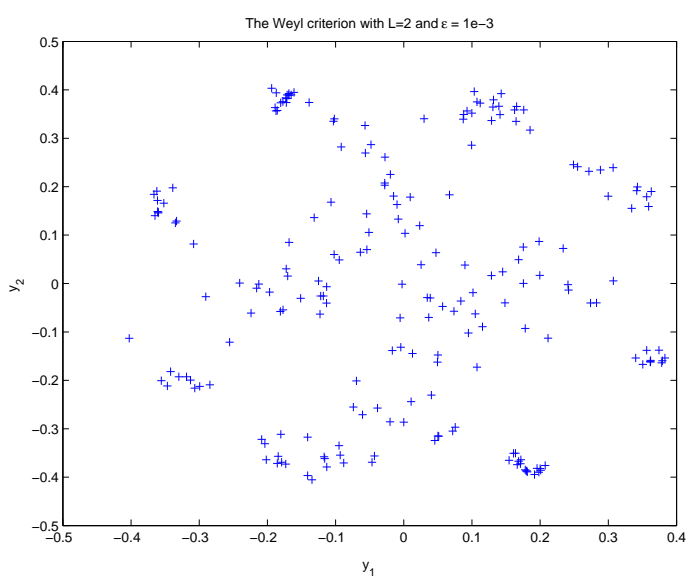

a. $\quad L=2$

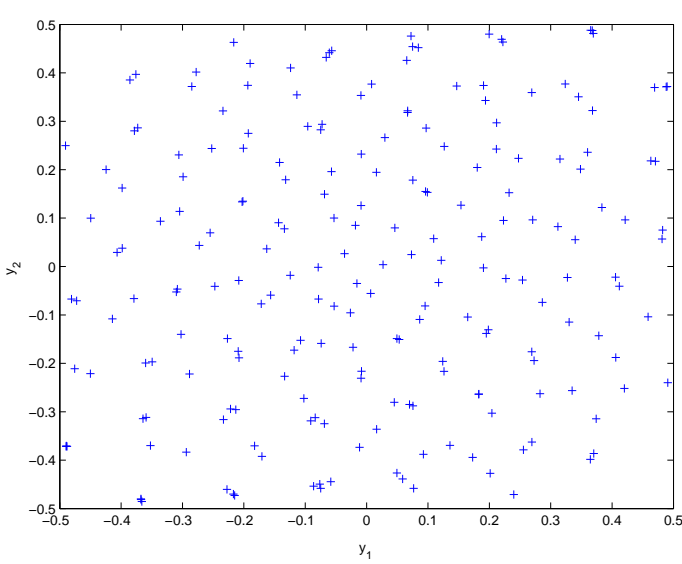

b. $L=6$

Figure 1. Output state space comparison for the Example problem, $L=2$ and 6 , $\epsilon=10^{-3}$

Pendse, G.: 2004, 'Optimization-based formulations using the Weyl criterion for input signal design in system identification'. Master's thesis, Arizona State University, Tempe, AZ, U.S.A.

Rivera, D., H. Lee, M. Braun, and H. Mittelmann: 2003, 'Plant-friendly system identification: a challenge for the process industries'. In: Proc. of the 13th IFAC Symposium on System Identification (SYSID 2003). Rotterdam, Netherlands, pp. 917-922.

Sriniwas, G. R., Y. Arkun, I.-L. Chien, and B. Ogunnaike: 1995, 'Nonlinear identification and control of a high-purity distillation column: a case study'. J. Proc. Cont. 5, 149.

Stenman, A.: 1999, 'Model on Demand: Algorithms, Analysis and Applications'. Ph.D. thesis, Dept. of Electrical Engineering, Linköping University, Sweden.

Weischedel, K. and T. McAvoy: 1980, 'Feasibility of decoupling in conventionally controlled distillation column'. Ind. Eng. Chem. Fund. 19, 379-384.

Weyl, H.: 1916, 'Über die gleichverteilung von zahlen mod eins'. Math. Ann. 77, 313-352. 
Table II. Results summary for signals designed for the Weischedel-McAvoy distillation column Case Study.

\begin{tabular}{cccccc}
\hline$C_{y_{1}}$ & $C_{y_{2}}$ & $\Delta C_{y_{1}}$ & $\Delta C_{y_{2}}$ & $\Delta C_{u_{1}}$ & $\Delta C_{u_{2}}$ \\
\hline 0.5 & 0.5 & 0.47 & 0.47 & 2.2 & 2.2 \\
\hline
\end{tabular}

\begin{tabular}{|c|c|c|c|c|c|c|}
\hline Type & Signal $(x)$ & $\mathrm{CF}(x)$ & PIPS(\%) & $\max \Delta x$ & $\max x$ & $\min x$ \\
\hline \multirow{4}{*}{$\begin{array}{l}\text { min CF }(u) \text { design; standard zippered } \\
\text { spectrum }\end{array}$} & $u_{1}$ & 1.21 & 82.43 & 0.0025 & 0.0020 & -0.0020 \\
\hline & $u_{2}$ & 1.22 & 81.77 & 0.0026 & 0.0020 & -0.0020 \\
\hline & $y_{1}$ & 2.48 & 48.84 & 0.0037 & 0.0325 & -0.0211 \\
\hline & $y_{2}$ & 2.19 & 46.12 & 0.0031 & 0.0199 & -0.0204 \\
\hline \multirow{4}{*}{$\begin{array}{l}\min \mathrm{CF}(y) \text { design; modified zippered } \\
\text { spectrum using NARX model } \\
\text { prediction }|\Delta u| \leq 0.01,|\Delta y| \leq 0.008 \\
\&|y| \leq 0.0085\end{array}$} & $u_{1}$ & 3.74 & 31.51 & 0.0100 & 0.0365 & -0.0254 \\
\hline & $u_{2}$ & 3.25 & 34.37 & 0.0100 & 0.0316 & -0.0250 \\
\hline & $y_{1}$ & 1.30 & 77.45 & 0.0051 & 0.0088 & -0.0086 \\
\hline & $y_{2}$ & 1.31 & 77.01 & 0.0082 & 0.0087 & -0.0086 \\
\hline \multirow{4}{*}{$\begin{array}{l}\text { data-centric experiment using NARX } \\
\text { model via a modified zippered } \\
\text { spectrum subject to }|\Delta u| \leq 0.01 \\
|\Delta y| \leq 0.08 \&|y| \leq 0.0085\end{array}$} & $u_{1}$ & 2.78 & 37.52 & 0.0079 & 0.0292 & -0.0268 \\
\hline & $u_{2}$ & 2.50 & 41.28 & 0.0076 & 0.0240 & -0.0225 \\
\hline & $y_{1}$ & 1.79 & 56.54 & 0.0062 & 0.0084 & -0.0082 \\
\hline & $y_{2}$ & 1.76 & 57.13 & 0.0053 & 0.0082 & -0.0083 \\
\hline
\end{tabular}

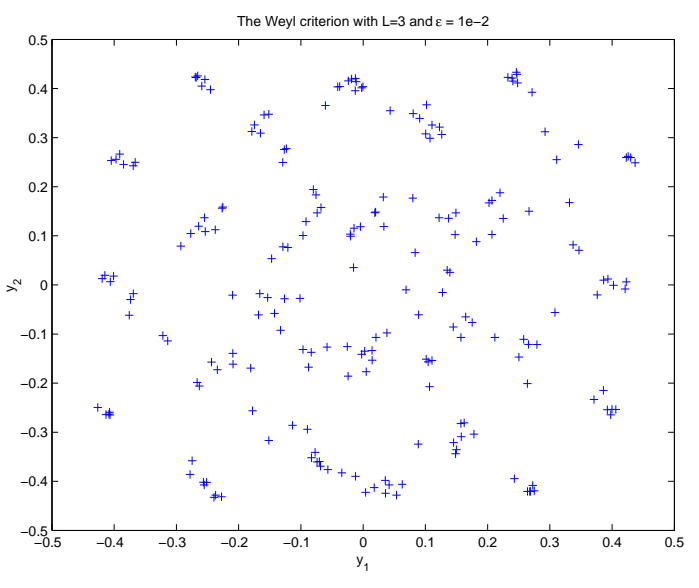

a. $\epsilon=10^{-2}$

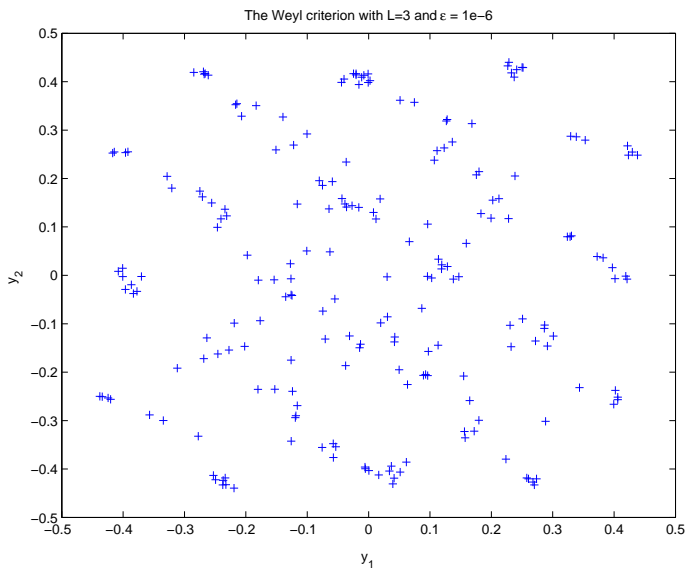

b. $\epsilon=10^{-6}$

Figure 2. Output state space comparison for the Example problem, $\epsilon=10^{-2}$ and $10^{-6}, L=3$ 


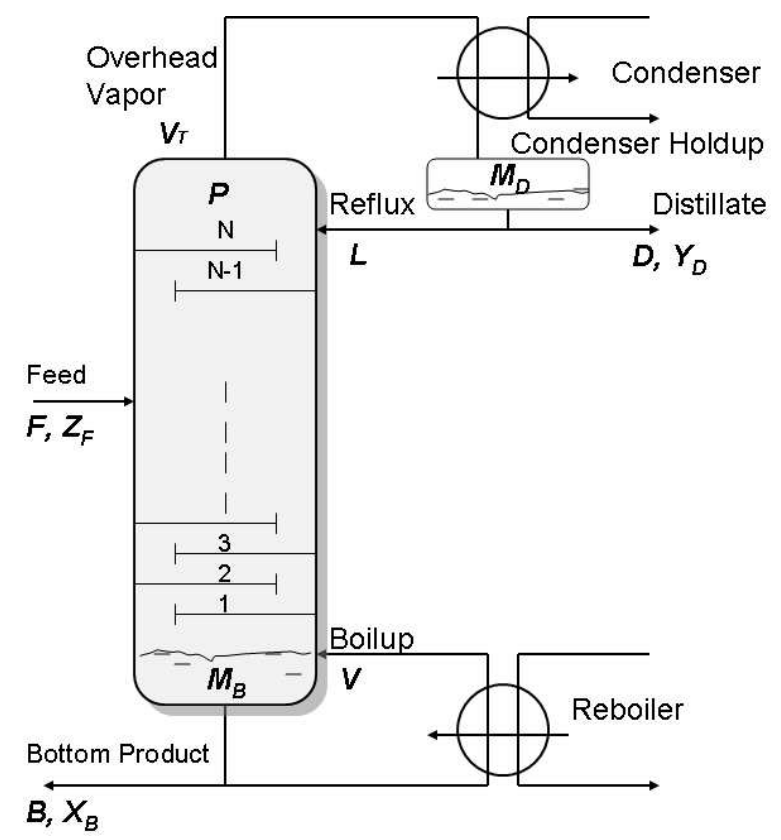

Figure 3. Binary distillation column schematic, per Morari and Zafiriou (1988)

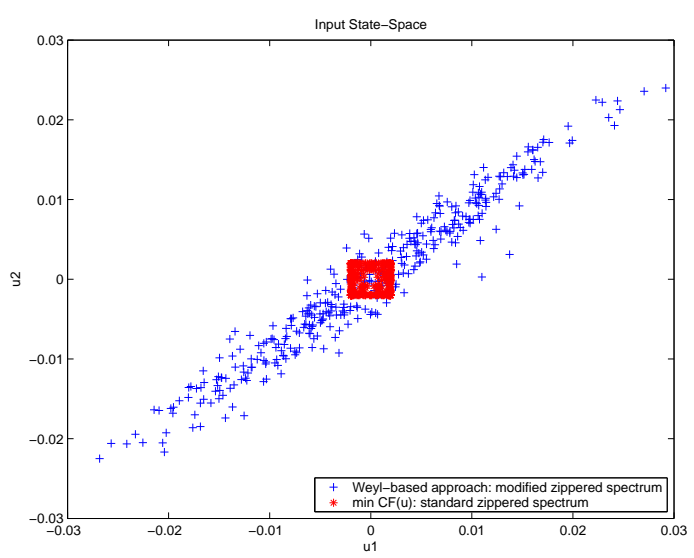

a. Input State-Space

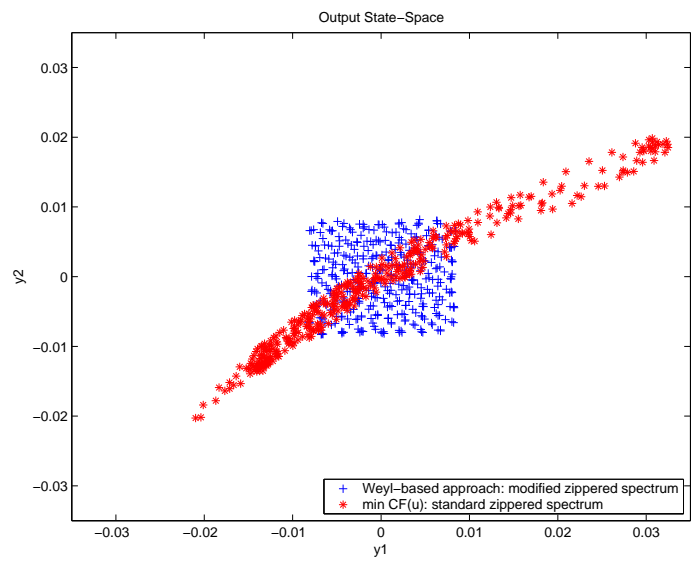

b. Output State-Space

Figure 4. Comparison of the input and output state-spaces for a standard "zippered" multisine $(*$, red $)$ vs. a Weyl-based signal design with modified spectrum $(+$, blue) for the Weischedel-McAvoy distillation column. 


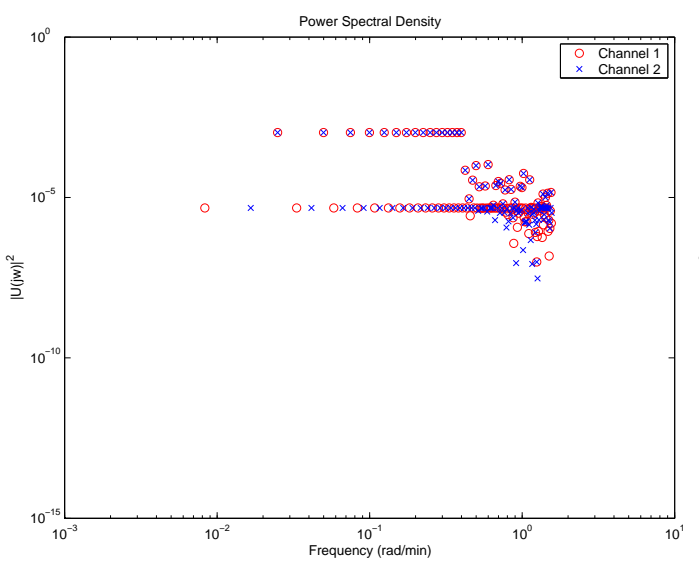

a. $\min \mathrm{CF}(y)$

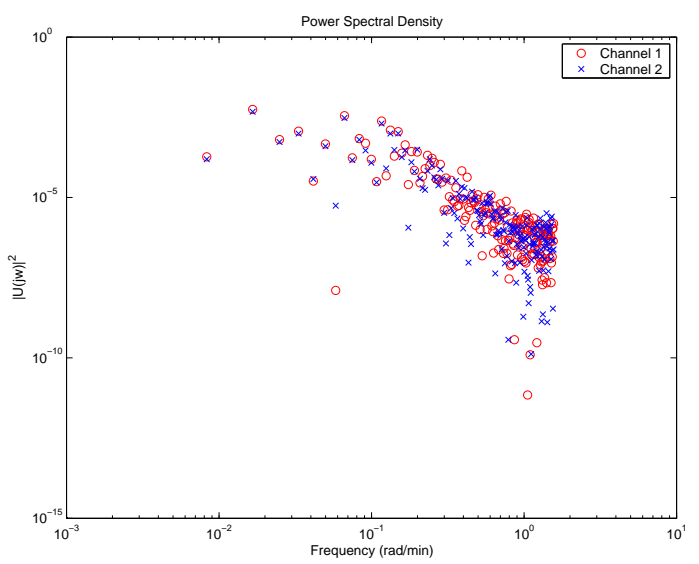

b. Weyl-based Approach

Figure 5. Input power spectral densities for Weischedel-McAvoy distillation column: min $\mathrm{CF}(y)$ modified zippered spectrum signal (a) versus Weyl-based design (b)

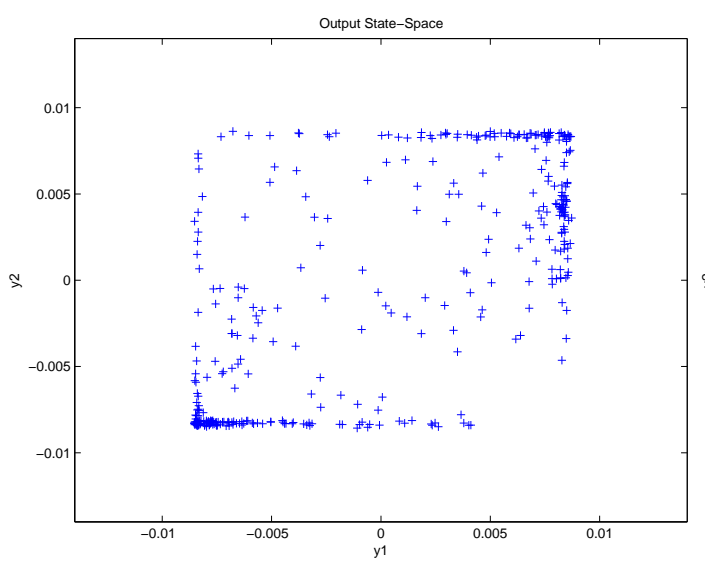

a. $\min \mathrm{CF}(y)$

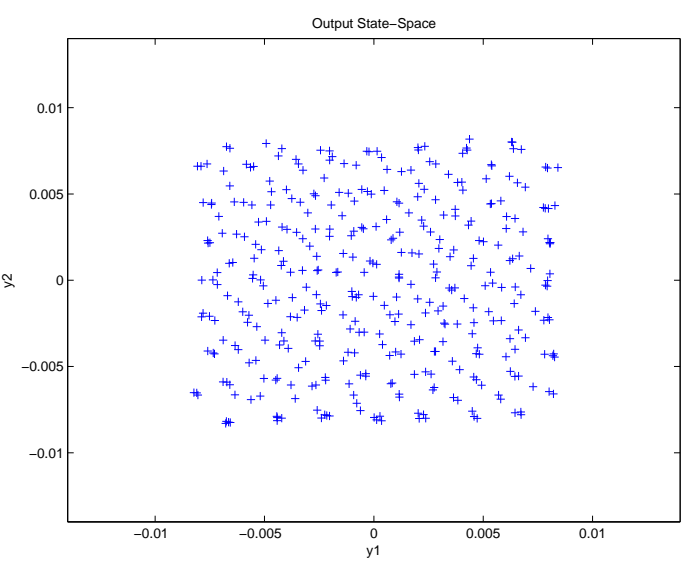

b. Weyl-based Approach

Figure 6. Output state-space analysis for Weischedel-McAvoy distillation column: min $\mathrm{CF}(y)$ modified zippered spectrum signal (a) versus Weyl-based design (b) 